\section{ROBÓTICA INCLUSIVA: RENDIMIENTO ECONÓMICO Y EMPLEO}

\author{
María Yolanda Sánchez-Urán Azaña \\ Universidad Complutense de Madrid \\ http://orcid.org/0000-0003-0030-8964 \\ sanuran@ucm.es
}

Cómo citar este artículo/Citation: Sánchez-Urán Azaña, María Yolanda (2021). Robótica Inclusiva: rendimiento económico y empleo. Arbor, 197(802): a626. https://doi.org/10.3989/arbor.2021.802004

Recibido: 1 marzo 2021. Aceptado: 20 diciembre 2021. Publicado: 3 febrero 2022.

RESUMEN: En el contexto actual los juristas están obligados a comprender el fenómeno de la digitalización. Para ello, deben primero, entenderlo, definirlo y delimitarlo y, luego, explicarlo y analizarlo para proponer soluciones justas y equitativas. Este concepto global abarca múltiples realidades, cada una con sus propias características y su impacto particular, en concreto en el ámbito del trabajo como fuente de identidad personal y social. Desde hace tiempo, instituciones públicas y privadas, internacionales, europeas y nacionales como la Organización para la Cooperación y el Desarrollo Económicos (OCDE), la Organización Internacional del Trabajo (OIT), el World Economic Forum (WEF) y la Unión Europea (UE), entre otras, realizan estudios, informes y análisis periódicos de la situación. En particular, y en lo que concierne a la Robótica, han traspasado el ámbito específico del sector industrial, para intentar identificar el impacto de tecnologías que replican en las máquinas procesos cognitivos similares a los humanos, desde una triple perspectiva: ética, legal y socioeconómica. Así, se hace hincapié en su proyección en la configuración y definición del trabajo; en los diferentes modelos o formas de trabajo, profundamente alterados hoy por la automatización o digitalización; y en las condiciones en las que las y los trabajadores prestan sus servicios. Desde esta triple perspectiva, admitiendo que los robots y los sistemas de inteligencia artificial (IA) son unos de los grandes inventos verdaderamente disruptivos del entorno digital y que han venido para quedarse, este estudio se centra en la Robótica Inclusiva, contribuyendo de este modo al entendimiento del cambio tecnológico actual al reflexionar sobre algunas de las cuestiones que este plantea para el Derecho.

PALABRAS CLAVE: automatización: robotización; inteligencia artificial; IA; empleo; igualdad.

\section{INCLUSIVE ROBOTICS: ECONOMIC PERFORMANCE AND EMPLOYMENT}

Copyright: (c) 2021 CSIC. Este es un artículo de acceso abierto distribuido bajo los términos de la licencia de uso y distribución Creative Commons Reconocimiento 4.0 Internacional (CC BY 4.0).

ABSTRACT: In the current context, jurists are obliged to understand the phenomenon of digitalisation. To do so, they must first understand, define and delimit it, and then explain and analyse it in order to propose fair and equitable solutions. This global concept encompasses multiple realities, each with its own characteristics and specific impact, in particular in the field of work as a source of personal and social identity. Public and private institutions, as well as international, European and national ones such as the OECD, ILO, WEF, EU, among others, have been doing regular studies, reports and analyses of the situation for some time. With regard to Robotics, they have gone beyond the specific scope of the industrial sector to try to identify the impact of technologies that replicate human-like cognitive processes in machines, seen from a triple perspective: ethical, legal and socio-economic. Thus, emphasis is placed on their effects on the configuration and definition of work; on the different models or types of work, profoundly altered today by automation or digitalisation; and on the conditions in which workers provide their services. From this triple perspective, admitting that robots and Artificial Intelligence systems are one of the great truly disruptive inventions of the digital environment and that they are here to stay, this study focuses on Inclusive robotics, thus contributing to an understanding of the current technological change by reflecting on some of the issues it raises for the law.

KEYWORDS: automation; robotization; Artificial Intelligence; $\mathrm{Al}$; employment; equality. 


\section{REFLEXIONES INICIALES}

¿Qué se entiende por Robótica Inclusiva y cuál es su proyección en el ámbito social?, ¿cómo impacta la digitalización en el mercado de trabajo?, ¿puede influir la robotización en la consecución de mercados de trabajo inclusivos?

Estas cuestiones sirven para abordar con garantías de éxito este debate. Contribuyen además a proponer soluciones éticas, fiables, para las empresas, la población trabajadora y la sociedad en su conjunto.

Las empresas buscan maximizar la productividad; la población trabajadora, la garantía de sus derechos; la sociedad en su conjunto conseguir la integración real y efectiva de la ciudadanía en el ámbito social y jurídico, sobre todo, a través del empleo y la ocupación. Desde el proyecto Inclusive Robotics for a better Society $^{1}$ se plantean los retos y oportunidades de la robótica en el marco legal, en especial, en el ámbito del empleo y la ocupación, así como sus proyecciones en el Derecho Social, Derecho del Trabajo y de la Protección Social, en especial, la Seguridad Social.

Desde hace tiempo, hay propuestas que plantean la colaboración, cooperación e interactuación de los seres humanos con los robots en el ámbito laboral, lo que está permitiendo pensar en retos y oportunidades en torno a este factor, uno de los más importantes para la integración efectiva y real de las personas en la sociedad; a saber, su trabajo. Para que estas propuestas sean viables y exitosas deben tener en cuenta varias dimensiones. No existe instantánea fija que abarque todos los sectores, todos los tipos de trabajo, todos los niveles de cualificación, todos los mercados y todos los países. Desde una perspectiva temporal, no cabe realizar proyecciones a largo plazo exclusivamente. Es necesario hacerlas también a corto y medio plazo asentando, en primer lugar, los retos $y$, en segundo lugar, las respuestas más adecuadas de consuno con el contexto y la realidad nacional concreta, pero también, en una dimensión más amplia, con la europea e internacional; sin olvidar la proyección funcional, los sectores de la actividad, las tareas desarrolladas por la población trabajadora y el grado de cualificación y habilidades para desarrollarlas.

Si atendemos a los factores descritos, la realidad no es homogénea desde lo temporal, espacial y funcional. En consecuencia, hay que promover acciones en las que la ausencia de limitaciones a la innovación tecnológica vaya de la mano del principio de que la automatización y la robótica deben permitir que el empleo se centre en los trabajos que añaden mayor valor. Esto implica un compromiso entre el desarrollo de competencias tecnológicas y el hacer frente a las necesidades que derivan del crecimiento y de la competitividad para minimizar las perturbaciones que la tecnología pueda generar en el mercado laboral y evitar las desigualdades sociales. Además, deben tratarse la brecha digital y el coste social que esta conlleva asociado, prestándose especial atención a la vulnerabilidad de determinados colectivos, entre otros, aquéllos que sufren discriminaciones por razón de género y edad. Por último, es necesario que exista una red de seguridad para el periodo de transición con propuestas de medidas políticas y legislativas al respecto.

La perspectiva jurídica aporta nuevas e interesantes aproximaciones conceptuales al desarrollo equitativo de la Robótica y a la inclusión de los robots en la sociedad. Aunque hay cierto consenso en las características que poseen estos dispositivos (hardware devices) para ser considerados robots ${ }^{2}$, su funcionalidad (diríamos multifuncionalidad) y el grado de interacción con los seres humanos permiten, en el estadio de desarrollo actual, adoptar una perspectiva más restrictiva orientada a la actividad y sector industrial y otra más amplia, para pensar los sistemas de inteligencia artificial (IA) implantados en robots, sobre todo cuando se trata de Robots de servicios, es decir, aquellos que se utilizan en la sanidad y en el cuidado de personas, que tienen por tanto una interacción intensa con los seres humanos.

Los problemas jurídicos relacionados con la IA y los robots trascienden a varias disciplinas o áreas de conocimiento dentro del Derecho, entre ellas, el tributario y financiero, el civil, el laboral y el relacionado con la seguridad social, aunque todas ellas confluyen en la

1 INBOTS, Proyecto Horizonte 2020, Unión Europea, No 780073. Puede consultarse en: http://inbots.eu/

2 En función de la capacidad de su software, se ha avanzado tanto estos años que resulta complejo describir con exactitud las características distintivas de los robots desde un punto de vista técnico. Erica Palmerini (2017:65) destacaba las siguientes: capacidad de recoger datos mediante sensores; procesar los datos en bruto; planificar y cumplir acciones mediante conocimientos e informaciones adquiridas, generalmente, en función de objetivos prefijados (descritas como sense-think-act). Como indicaba no hace mucho Juan García-Prieto la capacidad de comunicación con un operador con otros robots o con una red externa y la de aprendizaje, eran características eventuales (García-Prieto, 2018: 38). 
búsqueda de soluciones encaminadas a la proyección de los robots en el Mercado de Empleo. Para evitar que la robotización se traduzca en la sustitución de trabajos, tareas o actividades realizadas actualmente por los seres humanos o, incluso, en el reemplazo de las personas trabajadoras por máquinas más o menos inteligentes, se debe reflexionar sobre los principios, alcance y modalidades de intervención regulatoria que permitan salvaguardar los derechos humanos, es decir, aquellos derechos irrenunciables derivados de la dignidad humana.

Desde el año 2004, se habla de que la Roboética ${ }^{3}$ es el germen de los avances regulatorios que se han dado, en la actualidad, en países e instituciones, nacionales, europeas e internacionales, atendiendo a dos de sus cometidos más importantes. Por un lado, en definir criterios que sirvan para tratar los problemas que surgen con la fabricación y el uso de los robots (incluyendo los que afectan a las personas, usuarias o no); y, por otro lado, en aquellos relacionados con la ética de los robots, es decir, con las pautas de comportamiento ético que pueden ser introducidas en un robot o en la fusión humano/máquina (De Asís, 2014: 38). Como bien se ha afirmado, y los juristas debemos tenerlo muy cuenta, la ética necesita del Derecho para hacer más fuertes sus conclusiones y el Derecho necesita de la ética para fundamentar sus normas (De Asís, 2014: 38). Se requiere, por tanto, avanzar en una proyección de la Robótica sobre el mercado de trabajo triple, ética, jurídica y política, porque un aspecto sustancial de esa vocación de la Robótica queda representada por quienes hacen de su trabajo el principal factor de inclusión social y económica, en un contexto en el que el «ser humano está en el centro» [conforme a los informes últimos de la Organización Internacional del Trabajo (OIT) y de las instituciones europeas, entre otros la Propuesta de Reglamento del Parlamento Europeo y del Consejo por el que se establecen normas armonizadas en materia de Inteligencia Artificial ${ }^{4}$ y la Propuesta de Directiva sobre mejora de las condiciones de digitales ${ }^{5}$. Cuando hablamos de la apuesta, o fina- lidad, por un trabajo más humano (bajo el principio de human in command, en el que se fundamenta la propuesta de la UE sobre $I A^{6}$ ) estamos definiendo la robótica como inclusiva, como un instrumento para conseguir que así sea.

La robotización está afectando muy rápidamente al mercado laboral. Así, hay que centrarse en la forma en que este mercado podría cambiar para articular algunas soluciones que garanticen unos procesos de robotización socialmente responsables. Este hecho requiere, en primer lugar, partir del principio de innovación tecnológica, social y jurídicamente responsable, que combina la responsabilidad social de los actores implicados, especialmente de las empresas, con normas de obligado cumplimiento en garantía de los derechos fundamentales de las personas trabajadoras. No obstante, la regulación, en sentido más continental, no puede orientarse desde una perspectiva de defensa en contra de la innovación tecnológica en robótica, sino que debe articular medidas que den certeza a las partes implicadas con base en el principio antes mencionado.

Para ello, con fundamento en los derechos fundamentales previstos en nuestra Comunidad Europea (CE), y ahora también en la Unión Europea (UE), se requiere que los poderes públicos, junto a interlocutores sociales, promuevan una legislación que pondere y equilibre la garantía de la libertad de empresa (que incluye la libertad de innovación) con la de los derechos de las y los trabajadores ${ }^{7}$. Desde esta doble perspectiva, la legislación debe avanzar en garantizar la empleabilidad de las personas y en ponderar los incentivos para la puesta en marcha de actividades y empresas altamente tecnologizadas, evitando la pérdida de población trabajadora en sectores que están reconvirtiendo su modelo tradicional en otros más tecnologizados. En este sentido, se hace necesario introducir, al menos en la fase de transición, incentivos económicos para aquellas empresas que inviertan real y efectivamente en la capacitación tecnológica de sus trabajadores.

3 Este término ha sido acuñado por Gianmarco Veruggio (2006) y cabe definirlo como el conjunto de criterios o teorías para dar respuesta a los problemas éticos derivados del diseño, desarrollo y uso de los robots. Véase también: Gianmarco Veruggio y Fiorella Operto (2006, 2008).

4 Puede consultarse en https://eur-lex.europa.eu/legal-content/ES/PIN/?uri=CELEX:52021PC0206\#.

5 Puede consultarse en https://ec.europa.eu/commission/presscorner/detail/es/ip_21_6605

6 Véase Propuesta de Reglamento del Parlamento Europeo y del Consejo por el que se establecen normas armonizadas en materia de inteligencia artificial (Ley de Inteligencia Artificial). Véase nota 4.

7 El Pilar Europeo de Derechos Sociales ha establecido veinte principios para fortalecer una Europa social, justa e inclusiva, acompañados de un Plan de Acción hasta 2030. Ver: https://ec.europa.eu/info/strategy/priorities-2019-2024/economy-works-people/jobs-growth-and-investment/european-pillar-social-rights_es\#documents. 
Por otro lado, el Derecho del Trabajo ha de reflexionar sobre la posibilidad de que un robot inteligente pueda ser considerado como parte de la población trabajadora, es decir, con un estatus laboral reconocido, aunque no sea considerado jurídicamente como trabajador asalariado o por cuenta propia; el Derecho del Trabajo se fundamenta en el trabajo humano y el robot, aunque con altas dosis de IA, no puede ser considerado como tal. No obstante, son muchas las implicaciones o proyecciones de la robótica en la regulación, directa o indirecta, de las condiciones de trabajo, de las obligaciones y derechos, tanto del empresario como del trabajador.

El punto de partida del análisis es el principio de igualdad y de no discriminación; es decir, la igualdad real y efectiva de las personas, especialmente las más vulnerables a la automatización o robotización. De modo que ésta no puede convertirse, directa o indirectamente, en causa de discriminación. La neutralidad tecnológica no puede implicar desventajas o barreras para las personas. En este sentido, y también en la fase de transición, como medida excepcional, limitada y condicionada, debe regularse una cuota de personal humano en las empresas o medidas de ajuste razonable para aquellos colectivos especialmente vulnerables, entre ellos, los trabajadores de edad avanzada.

Como se desprende de esta primera reflexión, se debe tratar de adaptar las reglas y normativas laborales atinentes a los derechos y obligaciones de los y las trabajadoras. Esto implica, por un lado, garantizar la empleabilidad de las personas pensando más allá de los puestos de trabajo (lo que influirá en la deriva legislativa en torno al efecto sustitución-reemplazo de seres humanos por robots) y, por otro, garantizar los derechos de la población trabajadora ante la interactuación de trabajador-robot en el lugar del trabajo (cooperación entre ellos). Analizamos a continuación el contexto actual de la UE y nos centramos posteriormente en la Robótica Inclusiva y su proyección en el empleo y la ocupación, con propuestas al respecto.

\section{EL CONTEXTO ACTUAL EUROPEO. ÉTICA Y RES- PONSABILIDAD COMO TRASFONDO DE LAS RE- GLAS JURÍDICAS}

La Robótica, en especial la Robótica con IA, debe concebirse también como una innovación tecnológica para el ser humano, centrada en la persona y fundamentada en el compromiso de ser utilizada al servicio de la humanidad y del bien común, con el objetivo de mejorar el bienestar y la libertad de las personas. Esta visión, hoy consolidada, es sin duda, la perspectiva que están adoptando las instituciones de la UE, buscando el consenso y cooperación de todos los Estados miembros, con apuesta hacia una visión de esas tecnologías antropocéntrica, antropogénica y controlada por seres humanos. Así, se pretende el desarrollo, despliegue y uso de la Robótica de forma que se garantice en todo momento una supervisión humana integral, esto es, con control humano cuando sea necesario, incluso mediante la alteración o desactivación de dichas tecnologías.

Desde la publicación del informe del Parlamento Europeo con las primeras recomendaciones destinadas a la Comisión sobre normas de Derecho civil sobre robótica (Parlamento Europeo, 2017), se han elaborado diversos estudios que han orientado a las instituciones de la UE en sus directrices de soft law a través de comunicaciones y recomendaciones en las que instan a los Estados miembros a establecer medidas en torno a la digitalización y, en concreto, en lo que se ha convenido en denominar Economía Digitalizada. El objetivo en este contexto es profundizar en los retos y oportunidades de la IA, la robotización y las tecnologías conexas y regular su posible impacto en la economía y en la sociedad.

En todos estos documentos, se ha afrontado conjuntamente la digitalización, la IA y la robotización, desde una perspectiva que gira en torno a la ética y la responsabilidad. Tal vez en el convencimiento de que es muy difícil encontrar el consenso necesario para dictar normas de armonización y coordinación en el acervo comunitario integrado por reglas jurídicas precisas y de obligado cumplimiento por los Estados miembros, en unos casos, se han fijado mínimos para todos los ordenamientos jurídicos y, en otros, se han establecido reglas y medidas de coordinación. Cabe señalar que, a pesar de estos intentos de regulación común, en la actualidad, existe una maraña de disposiciones de soft law en las que se aprecian orientaciones, guías, recomendaciones que, a veces, no son totalmente armónicas ni siguen un patrón claro, de propuestas y objetivos.

La transición hacia una economía digitalizada es una de las principales preocupaciones en los trabajos de organizaciones internacionales, nacionales, y también instituciones de la UE; en ellos existe un intenso debate en torno a qué y cómo regular en relación con ese fenómeno de generalización de la robotización, más allá del inicialmente considerado ámbito industrial. 
Y aunque aún está por definir la conexión entre soft law o autorregulación y un pilar mínimo de normas jurídicas imperativas, de prohibición o de promoción, la orientación actual incide en la primera dimensión, soft law. Así lo reflejan los últimos documentos elaborados por las instituciones de la UE, la Comisión Europea y el Parlamento Europeo. Sin embargo, desde mediados de 2021 se ha avanzado en propuestas legislativas que en estos momentos se encuentran en fase de elaboración. Estos textos se han trabajado paralelamente para conformar lo que puede denominarse el paquete de la UE sobre IA, orientado hacia lo que cabe entenderse como una IA para Europa, definida como ética, fiable y responsable.

La Comisión Europea adoptó en 2018 la Comunicación sobre inteligencia artificial para Europa, que dirige a las otras instituciones de la UE como el Parlamento, el Consejo, el Comité Económico y Social y el Comité de las Regiones (COM/2018/237 final/2) ${ }^{8}$. Este texto sirvió de base al Grupo Independiente de Expertos de alto nivel sobre IA, nombrado por la Comisión Europea en junio de 2018, para elaborar el documento Directrices éticas para una IA Fiable, publicado en 2019, que usó también la Comisión Europea para elaborar el Libro Blanco sobre la inteligencia artificial: un enfoque europeo orientado a la excelencia y la confianza publicado en 2020 (Grupo Independiente de Expertos de alto nivel sobre IA, 2019; Comisión Europea, 2020).

Por otro lado, el Parlamento Europeo presentó en 2019 su resolución sobre una política industrial global europea en materia de inteligencia artificial y robóti$\mathrm{ca}^{9}$ donde abogaba porque ambas sean transparentes e integren consideraciones éticas para enriquecer la vida y consolidar las capacidades humanas. Examinaba y comparaba en detalle las diferentes directrices que a nivel nacional habían afrontado los Estados miembros en el desarrollo y uso de IA y robótica, destacando las lagunas detectadas, así como las implicaciones más complejas y menos seguras de la inteligencia artificial. En este sentido, utilizaba la definición de IA acuñada por la Comisión Europea en su comunicación COM (2018)0237 (véase nota 8) y hablaba de consideraciones éticas, sociales y jurídicas asociadas a los robots, considerados como robots inteligentes, es decir aquellos que llevan incorporado un software que les hace no solo más automáticos, sino más autónomos. Se avanza así desde tecnologías basadas en reglas (rules-based technologies) a las tecnologías basadas en la predicción (prediction-based technologies). Las primeras son tecnologías que automatizan las tareas codificándolas en una serie de afirmaciones "si-entonces" que se incorporan al software. Como tales, estas tecnologías pueden automatizar tareas que siguen un protocolo determinado, tareas que a veces se denominan "tareas rutinarias». Ejemplos de estas tareas rutinarias (o codificables) son realizar cálculos y ensamblar productos en una cadena de montaje. Las tecnologías basadas en la predicción utilizan técnicas de big data y aprendizaje automático para un uso predictivo, e incluso prescriptivo, de los resultados probables (Inbots, Informe Preliminar, 2019.41) ${ }^{10}$.

Entre las cuestiones sociales que se trataban en el informe, estaban los posibles efectos que la IA podía tener en el mercado laboral, centrándose en el probable impacto en el crecimiento económico, la productividad y en la fuerza de trabajo y en los posibles efectos que podía ocasionar en diferentes demografías, como el empeoramiento de la brecha digital. Además, reflexionaba sobre las consecuencias del despliegue de la IA en los centros de trabajo y consideraba el impacto potencial de la IA sobre la desigualdad, aunque también planteaba cómo los beneficios de la IA podrían ser compartidos dentro de la sociedad. Este informe fue fundamento de dos Resoluciones del Parlamento Europeo publicadas el 20 de octubre de 2020: la Resolución del Parlamento Europeo, de 20 de octubre de 2020, con recomendaciones destinadas a la Comisión sobre un marco de los aspectos éticos de la inteligencia artificial, la robótica y las tecnologías conexas ${ }^{11}$ y el Informe con recomendaciones destinadas a la Comisión sobre un régimen de responsabilidad civil en materia de inteligencia artificial (Parlamento Europeo, 2020).

El primer documento, la Resolución del Parlamento Europeo, de 20 de octubre de 2020, con recomenda-

8 Comunicación de la Comisión al Parlamento Europeo, al Consejo, al Comité Social y Económico Europeo y al Comité de las Regiones sobre Inteligencia Artificial para Europa. COM/2018/237 final/2. Disponible en: https://ec.europa.eu/transparency/documents-register/detaiI?ref=COM(2018)237\&lang=es.

9 Resolución del Parlamento Europeo, de 12 de febrero de 2019, sobre una política industrial global europea en materia de inteligencia artificial y robótica (2018/2088(INI)). Disponible en: https://www.europarl.europa.eu/doceo/document/TA-8-2019-0081_ES.html

10 Este informe está disponible en la página web del proyecto INBOTS: http://inbots.eu/.

11 Disponible en: https://www.europarl.europa.eu/doceo/document/TA-9-2020-0275_ES.html [última actualización: 22 de enero de 2021]. 
ciones destinadas a la Comisión sobre un marco de los aspectos éticos de la inteligencia artificial, la robótica y las tecnologías conexas (véase nota 11), incorporaba como anexo una propuesta de reglamento sobre los principios éticos para el desarrollo, el despliegue y el uso de la inteligencia artificial, la robótica y las tecnologías conexas, cuyas definiciones han guiado esta investigación:

«inteligencia artificial»: «un sistema basado en programas informáticos o incorporado en dispositivos físicos que manifiesta un comportamiento inteligente al ser capaz, entre otras cosas, de recopilar y tratar datos, analizar e interpretar su entorno y pasar a la acción, con cierto grado de autonomía, con el fin de alcanzar objetivos específicos». Se adapta por tanto la definición de la Comunicación de la Comisión Europea COM (2018)0237 (véase nota 8) y es la definición que se propone por la institución europea en la Propuesta de Reglamento del Parlamento Europeo y del Consejo por el que se establecen normas armonizadas en materia de Inteligencia Artificial (véase nota 4).

«autonomía»: «un sistema de inteligencia artificial que funciona interpretando determinados datos de entrada y utilizando un conjunto de instrucciones predeterminadas, sin limitarse a ellas, a pesar de que el comportamiento del sistema esté orientado a cumplir el objetivo que se le haya asignado y esté limitado por este y por otras decisiones de diseño pertinentes tomadas por su desarrollador». Se trata de distinguir ente automation y automatization; entre convertir a una máquina o a un dispositivo en un sistema más automático a otro más autónomo, operando por sí sino con capacidades tecnológicas más avanzadas.

«robótica», se define a partir del concepto de robots industriales de la norma ISO 8373 como «las tecnologías que permiten que las máquinas controladas automáticamente, reprogramables y multifuncionales realicen en el mundo físico acciones tradicionalmente realizadas o iniciadas por los seres humanos, en particular mediante la inteligencia artificial o las tecnologías conexas».

El Informe con recomendaciones destinadas a la Comisión sobre un régimen de responsabilidad civil en materia de inteligencia artificial, adoptaba la forma de un decálogo de diez principios regulatorios de la IA en la UE (Parlamento Europeo, 2020). Además, men- cionaba la responsabilidad social y los derechos sociales y laborales de las personas trabajadoras, aunque no adoptaba una definición de responsabilidad social, ni se especificaba si es un concepto diferente al de ética y al de cumplimiento de obligaciones jurídicas. La responsabilidad social se une a la igualdad de género y se muestran en esta resolución como principios fundamentales de la propuesta de reglamento sobre Inteligencia Artificial.

\section{HACIA UN ROBÓTICA INCLUSIVA EN EL EM- PLEO: LA RED DE SEGURIDAD PARA LAS PERSO- NAS TRABAJADORAS}

La Resolución del Parlamento Europeo, de 20 de octubre de 2020, con recomendaciones destinadas a la Comisión sobre un marco de los aspectos éticos de la inteligencia artificial, la robótica y las tecnologías conexas, arriba citada (véase nota 8), dedicaba un apartado a lo que denominaba «empleo, derechos de los trabajadores, competencias digitales y lugar de trabajo" y establecía diecisiete recomendaciones (de la 109 a la 116) que, en nuestra opinión, están desordenadas, son reiterativas y, en algún caso, también pueden considerarse inconexas. En primer lugar, no incorporaba una reflexión sobre el crecimiento económico, la productividad y el empleo o mercado de trabajo cuando, precisamente unos meses antes, el Parlamento Europeo, en su resolución de 12 de febrero de 2019 sobre política industrial global en materia de IA y Robótica ${ }^{12}$, había subrayado que la automatización unida a la inteligencia artificial aumentará la productividad y con ella la producción; y que, al igual que en anteriores revoluciones tecnológicas, se sustituirán algunos puestos de trabajo, pero también se crearán otros nuevos que transformarán nuestras vidas y prácticas profesionales. Además, indicaba que un mayor uso de la robótica y la inteligencia artificial también debería estar encaminado a reducir la exposición de los seres humanos a condiciones perjudiciales y peligrosas, así como a contribuir a crear más puestos de trabajo dignos y de calidad que mejoren la productividad. La Resolución citada también recogía algunos aspectos relacionados con el impacto en el empleo.

La conexión entre productividad (aumento por el desarrollo y uso de la IA y la robótica)-empleo (aplicación de la IA y la robótica para contribuir a mercados laborales inclusivo) -trabajo de calidad y digno,

12 Resolución del Parlamento Europeo, de 12 de febrero de 2019, sobre una política industrial global europea en materia de inteligencia artificial y robótica (2018/2088(INI)). Disponible en: https://www.europarl.europa.eu/doceo/document/TA-8-2019-0081_ES.html [Última actualización: enero 2021]. 
exige partir de unos presupuestos que marquen las acciones en torno a otros aspectos derivados. Una de las cuestiones fundamentales es analizar si el aumento de la productividad y la competitividad de las empresas (que no parece estar en duda) irá también acompañado de un aumento de la cantidad y calidad del empleo humano. En este sentido, se plantean dos problemas básicos relacionados con la desigualdad que pudiera derivar de la robotización: el efecto sustitución de los seres humanos por robots y la afectación de las condiciones de trabajo de los humanos, es decir, de los salarios, sobre todo de la población trabajadora menos cualificada, la evolución hacia la descentralización de la producción, la redistribución de los puestos de trabajo y de las tareas y el efecto del desempleo tecnológico.

La citada resolución sobre un marco ético ya citada (véase nota 8), utiliza expresiones que no tienen un significado cierto o que no constituyen una definición consensuada que sirva de base para las reflexiones y propuestas siguientes. Por ejemplo, no queda claro qué significado se da a la expresión poner al ser humano en el centro o cuando se señala que la IA y la Robótica deben estar centrada en el ser humano pues ha de tenerse en cuenta que el ser humano lo es desde múltiples perspectivas, como cliente, como ciudadano, como contribuyente, como trabajador.

Teniendo en cuenta solo la concepción humana relativa al ámbito laboral, la UE se hace eco del informe Trabajar para un futuro más prometedor elaborado por la OIT (2019). En él se propone un programa centrado en las personas para que el futuro del trabajo fortalezca el contrato social: sitúa a las personas y al trabajo que realizan en el núcleo de las políticas económicas y sociales y de la práctica empresarial. Este programa se asienta en tres ejes de actuación que, combinados entre sí, generarían crecimiento, igualdad y sostenibilidad para las generaciones presentes y futuras. Se centra, además, en la inversión (social y económica) en torno a las capacidades de las personas, las instituciones del trabajo y en el objetivo de un trabajo decente y sostenible, diríamos un trabajo más justo y equitativo. Desde esta perspectiva, por tanto, hay que entender la recomendación de que la IA, la robótica y las tecnologías conexas en el lugar del trabajo, es decir, en el ámbito del empleo y de la ocupación, debe orientarse hacia la consecución de un mercado laboral inclusivo. Y la razón es obvia, uno de los problemas éticos que se plantean en el área social gira en torno al trabajo (Lin, Abney y Bekey, 2011).

$Y$ aunque no se defina, debe entenderse que hay una llamada a los poderes públicos y a las instituciones privadas para que conjuntamente se responsabilicen de que las personas trabajadoras vean satisfecha la integración que exige un Estado Social. Así, la Robótica debe procurar que la innovación social y jurídicamente responsable se adopte pensando en que no puede orientarse jamás al reemplazo del trabajo humano (otra cosa, diferente es el cambio o reemplazo de tareas y habilidades) sino a facilitar la transición de determinadas tareas hacia otras más propias de los seres humanos, (aquellas denominadas habilidades sociales y emocionales, entre ellas, las avanzadas de comunicación y negociación; las de liderazgo y gestión o las orientadas a la enseñanza y a la formación). Esto, como veremos, requerirá un mayor compromiso social para preparar con éxito a la población trabajadora para este cambio, que la pandemia de la COVID-19 ha acelerado, en particular en los sectores más vulnerables: trabajadores y trabajadoras jóvenes con salarios bajos, mujeres e incluso grupos étnicos minoritarios (Lund et al., 2021). Así, será necesario identificar las profesiones que tienen más probabilidades de cambiar en un futuro próximo y las nuevas competencias que será necesario aprender.

\subsection{Impacto positivo en el contexto del respeto de los valores de la UE, los derechos humanos y los de- rechos fundamentales}

Términos tales como "valores», "derechos humanos», "derechos fundamentales» se utilizan a veces indistintamente en la resolución del Parlamento sobre un marco ético, mencionada previamente (véase nota 8), como si tuvieran el mismo significado o encerraran significados intercambiable ${ }^{13}$. Sin embargo, debe recordarse que su significado es diferente en el ámbito del Derecho. Tampoco se especifica en dicha Resolución qué valores, derechos humanos o derechos fundamentales y de qué manera pueden verse perjudicados en lo que la UE llama la transición, sin especificar

13 Véase también OCDE, Principios de la IA. En torno al que expresa como valores y equidad centrados en el ser humano, dice: «Los actores de la IA deben respetar el estado de derecho, los derechos humanos y los valores democráticos durante todo el ciclo de vida del sistema de IA. Estos incluyen libertad, dignidad y autonomía, privacidad y protección de datos, no discriminación e igualdad, diversidad, equidad, justicia social y derechos laborales reconocidos internacionalmente». Puede consultarse en https://www.oecd.ai/ai-principles (última visita 22 de febrero de 2021). 
de dónde o hacia dónde, o si es de la transición de unas tareas o puestos de trabajo a otros. Tampoco se explica si se hace referencia al proceso de sustitución o al proceso de transformación. En este sentido, la respuesta global que solicita el Parlamento Europeo a nivel de la UE debe provenir de la conjunción entre una productividad equilibrada y un progreso tecnológico equitativo, atendiendo a la garantía de los derechos en el empleo y en la ocupación

La innovación tecnológica social y jurídicamente responsable exige, en el sector del ordenamiento jurídico que lo disciplina, el Derecho Social, o más concretamente el Derecho del Trabajo y de la Protección (Seguridad) Social, el respeto de los derechos humanos asentados tanto en el acervo europeo (en los Tratados y la Carta de Derechos Fundamentales de la UE) como en el internacional (fundamentalmente a través de Convenios y Recomendaciones de la OIT) y en el ámbito interno español (con la Constitución Española al fondo) $)^{14}$. Así lo indica el Grupo Independiente de Expertos de alto nivel sobre IA en su informe Directrices éticas para una IA fiable: "El respeto de los derechos fundamentales, dentro de un marco de democracia y estado de Derecho, proporciona la base más prometedora para identificar los principios y valores éticos abstractos que se pueden poner en práctica en el contexto de la IA» (Grupo Independiente de Expertos de alto nivel sobre IA, 2019: 12).

Es muy importante analizar estas cuestiones, estas implicaciones ético-jurídicas, porque, lejos de reproducir lo que ya es habitual y aceptado por la ciudadanía y menos aún de reiterar lo que puede quedar como un mero repertorio de obviedades, es necesario atribuir un significado certero a la que pudiera denominarse teoría de los derechos humanos desde una innovación tecnológica, en particular la Robótica social y jurídicamente responsable. En efecto, atendiendo a lo que hemos afirmado en páginas anteriores, el progreso tecnológico y la innovación tecnológica, no tienen por qué estar reñidos con la mejora de la vida humana en general y, en particular, con su dimensión social, si este logra la responsabilidad intergeneracional, interpersonal.

En este contexto, ¿qué debemos entonces entender por dignidad humana, igualdad, no discriminación y solidaridad? Diferentes informes y estudios auspiciados por las instituciones de la UE hacen referencia a estos valores, principios y derechos.

Sobre la «dignidad humana» el Grupo Independiente de Expertos de Alto Nivel sobre IA designado por la Comisión Europea dice:

"La dignidad humana contiene en sí la idea de que todo ser humano posee un "valor intrínseco" que jamás se debe menoscabar, poner en peligro ni ser objeto de represión por parte de otros (ni de las nuevas tecnologías, como los sistemas de IA). En el contexto de la inteligencia artificial, el respeto de la dignidad humana implica que todas las personas han de ser tratadas con el debido respeto que merecen como sujetos morales, y no como simples objetos que se pueden filtrar, ordenar, puntuar, dirigir, condicionar o manipular. En consecuencia, los sistemas de IA deben desarrollarse de un modo que respete, proteja y esté al servicio de la integridad física y mental de los seres humanos, el sentimiento de identidad personal y cultural y la satisfacción de sus necesidades esenciales» (Grupo Independiente de Expertos de alto nivel sobre IA, 2019: 13).

Así, consideran que la dignidad humana está asentada como principio general de nuestro ordenamiento jurídico y sustentador de todos los derechos fundamentales. No tiene un carácter presuntamente abstracto y universal, sino que ha de concretarse en cada ser humano, atendiendo a su diversidad y diferencia, con el trasfondo de la igualdad real y efectiva, y a la libertad, que nos ayuda a un enfoque abierto, y equilibrado, hacia las nuevas tecnologías (De Asís, 2014: 61).

Desde la perspectiva del trabajo, del trabajo humano, la teoría del Derecho Social considera que la persona trabajadora tiene reconocido y el empresario ha de respetar la consideración a su dignidad. Pero, quizá cabe cuestionarse si el avance de la ciencia y de la tecnología afecta a la dignidad de la persona trabajadora también en su ámbito de trabajo. Como expresa Montoya Melgar, la dignidad es un concepto de raíz ética que el Derecho acoge. En la relación laboral, el empresariado ha de respetar «la consideración debi-

14 El acervo de la UE, que comprende la base común de derechos y obligaciones que vincula al conjunto de los países miembros de la UE, está en evolución constante. Engloba el contenido, los principios y los objetivos políticos de los Tratados; la legislación adoptada en aplicación de los Tratados y la jurisprudencia del Tribunal de Justicia de la UE; declaraciones y resoluciones adoptadas en el marco de la UE; los actos en materia de política exterior y de seguridad común; los actos acordados en materia de justicia y asuntos de interior; los acuerdos internacionales celebrados por la UE y los celebrados por los países de la UE entre sí en el ámbito de las actividades de la UE. 
da a la dignidad» de la persona trabajadora (art. 4.2, Estatuto de los Trabajadores). Además, está protegido frente a todo tipo de discriminación y, por tanto, «la conducta del empresario que no respete tal dignidad y suponga una actitud vejatoria o depresiva para el trabajador, da derecho a éste a optar entre solicitar la extinción del contrato de trabajo, percibiendo una indemnización equivalente a la que correspondería en caso de despido improcedente o solicitar del Juzgado de lo Social que anule la decisión lesiva del derecho fundamental y ordene al empresario de abstenerse de tales actos» (Montoya Melgar, 2020: 12).

Así, la innovación tecnológica y robótica social y jurídicamente responsable exige a quienes están implicados, que consideren la dignidad humana, asentada en ese principio recogido en las grandes Declaraciones Universales de Derechos Humanos, en la propia Constitución de la OIT y en nuestro Estado Social y Democrático de Derecho como uno de los fundamentos del orden político y de la paz social. Por ello, debe existir un criterio general de carácter interpretativo o «un valor espiritual y moral inherente a la persona, que se manifiesta singularmente en la autodeterminación consciente y responsable de la propia vida y que lleva consigo la pretensión al respeto por parte de los demás» (Sentencia del Tribunal Constitucional 181/2004), que garantice un "trabajo decente» (en términos de la OIT) o un trabajo digno, que permita el desarrollo de las propias capacidades, con respeto a los principios y derechos laborales fundamentes, que permita un ingreso justo al esfuerzo realizado, sin discriminación, y que se lleve a cabo con protección social.

Sobre la «igualdad», «no discriminación» y «solidaridad» el Grupo Independiente de Expertos de Alto Nivel sobre IA dice:

«Es preciso garantizar por igual el respeto del valor moral y la dignidad de todos los seres humanos. Este requisito va más allá de la no discriminación, que tolera el establecimiento de distinciones entre situaciones diferentes sobre la base de justificaciones objetivas. En el contexto de la IA, la igualdad implica que el funcionamiento de este tipo de sistemas no debe generar resultados injustamente sesgados (por ejemplo, los datos utilizados para la formación de los sistemas de IA deben ser lo más inclusivos posibles, de forma que estén representados los diferentes grupos de población). Esto también requiere un adecuado respeto de las personas y grupos potencialmente vulnerables, como los trabajadores, las mujeres, las personas con discapacidad, las minorías étnicas, los niños, los consumidores $u$ otras personas en riesgo de exclusión» (Grupo Independiente de Expertos de Alto Nivel sobre IA, 2009: 13).

La "solidaridad» es un valor que define a todo Estado Social y, en este sentido, aunque los grandes tratados y Constituciones como la española no recojan una definición exacta, debe recordarse que los principios rectores de la política social y económica se asientan en dicho valor desde la perspectiva territorial, personal e intergeneracional. La solidaridad se exige a unos territorios para con otros; a unos sujetos para con otros; a una generación para con otra. En concreto, se debe presuponer la solidaridad de los nativos digitales respecto de las generaciones anteriores, más analógicas. Aunque la solidaridad es un valor no definido de forma precisa, junto a la dignidad humana, ha de considerarse como base y sustento de otros derechos como los sociales: aquellos relacionados con el empleo, la ocupación y la protección social, elementos integrantes de la consecución de una igualdad real y efectiva en la que se asientan los derechos prestacionales (que exigen de los poderes públicos acciones para su implementación efectiva). No hay, por cierto, mayor solidaridad que la puesta en práctica por los poderes públicos de medidas tendentes a lograr la integración social y económica de los ciudadanos, incluidos los trabajadores.

Por último, planteamos el significado que la vertiente jurídica debe otorgar a la «igualdad» y la «no discriminación» respecto a la innovación tecnológica social y jurídicamente responsable.

Desde esa perspectiva jurídica, la «igualdad» es un principio, valor y derecho que tiene un significado cierto y encierra un mandato efectivo en todos los ámbitos de la vida social y económica, incluido por tanto el del trabajo. Como derecho tiene una doble perspectiva: la formal, todas las personas somos iguales y la material, no discriminación. Esto exige que los poderes públicos adopten medidas que permitan una integración de la ciudadanía real y efectiva, sobre todo, de las personas que, por determinadas circunstancias históricamente arraigadas, se ubican en colectivos que han padecido una situación de desigualdad en los diferentes ámbitos de la vida social incluido, por supuesto, el laboral. Es así como en los distintos ordenamientos jurídicos, también en el español y en el de la Unión Europea, se ha avanzado en la búsqueda de la igualdad y la eliminación de la desigualdad, prohibiendo la discriminación y adoptando incluso 
medidas de acción positiva para que los colectivos más vulnerables logren la integración efectiva en la sociedad. La diversidad no reemplaza a la igualdad, sino que ésta debe tenerla en cuenta para evitar que perviva la discriminación de colectivos especialmente desfavorecidos.

Pero la discriminación, pese a lo afirmado por el Grupo Independiente de Expertos de Alto Nivel sobre IA, no admite la diferencia de trato entre situaciones diferentes, pues está limitada por la igualdad formal. La «no discriminación» entronca con la igualdad real y efectiva y, por tanto, persigue acabar con las diferencias de trato perjudiciales que sufren históricamente algunas personas como las mujeres y las personas con diversidad funcional. $Y$ debe fijarse con precisión si la automatización produce o no desigualdad y en qué términos, o si, por el contrario, contribuye al crecimiento inclusivo.

En el ámbito de la automatización y la robotización, es necesario reflexionar sobre la relación o conexión entre seres humanos-robots, pero no como resultado de la comparación de unos seres humanos con otros atendiendo a las causas o razones históricas de desigualdad, sino comparando a los seres humanos con los robots para evitar lo que se conoce como automation anxiety, es decir, miedo a ser reemplazado por máquinas, inteligentes o no. Como muestran diversos estudios, este fenómeno es cíclico, emerge periódicamente en el debate público y parte de una interpretación no correcta de algunos trabajos académicos de comienzos de la segunda década del s. XXI.

Jose Ignacio López-Sánchez, Jose Luis Arroyo-Barrigüete y Tomás Curto-González, concluyen, como otros trabajos recientes, que «mayores niveles de automatización llevan a una reducción de la desigualdad a medio plazo» (López-Sánchez, Arroyo-Barrigüete y Curto-González, 2020: 172). Señalan, que la explicación a este fenómeno puede encontrarse en el hecho de que el aumento de la robotización genera una mayor riqueza en el país, que puede ser utilizada por los gobiernos para reducir la desigualdad a través de políticas redistributivas. Aceptando como cierta esta propuesta, desde la perspectiva del ámbito del trabajo, no debe dejarse de plantear en qué sentido puede crearse desigualdad y si la misma proviene del temido efecto sustitución humanos-robots y de la consideración que haya de darse a éstos en el ámbito del empleo y de la ocupación. Por ello, es necesario, promover medidas preventivas y no sólo reparadoras (distributivas), en el diseño de las políticas públicas/privadas.

Desde esta perspectiva orientada hacia la prevención, jurídicamente no puede hablarse de robots trabajadores y no cabe otorgarles un estatus laboral. El Derecho del Trabajo permite adoptar una posición crítica respecto de cualquier intento relacionado con la personalidad robótica o ente capaz no personal (ECNP), esto es, respecto del reconocimiento jurídico de esa nueva categoría de entes capaces. La voluntariedad y la consciencia no es algo que deban alcanzar los robots $y$, por tanto, permanecerían alejados de una relación laboral de carácter personal y patrimonial. Los robots, deben ser un instrumento de trabajo o una herramienta tecnológicamente compleja, sin derechos y obligaciones laborales debido a la ausencia de la voluntariedad.

Partiendo de esa consideración previa, se analizan a continuación determinados aspectos jurídicolaborales.

\subsection{Sobre las condiciones de trabajo y derechos bá- sicos de las personas trabajadoras}

En los documentos de las instituciones de la UE se indica que la IA y la robotización deben tener un impacto positivo en las condiciones de trabajo. Sin embargo, aunque es un objetivo loable, no queda claro a qué condiciones de trabajo nos estamos refiriendo o de qué manera la robotización puede incidir en esas condiciones de trabajo. Tampoco qué efecto puede generar un cambio de modelo de trabajo o una nueva polarización entre trabajadores y trabajadoras ni cuál es o cuáles son las medidas necesarias para conseguir ese impacto positivo.

Las instituciones de la UE indican que la IA y la robotización no deben afectar en modo alguno al ejercicio de los derechos fundamentales reconocidos en los Estados miembros y a escala de la Unión, incluido el derecho o la libertad de huelga o de emprender otras acciones contempladas por los sistemas de relaciones laborales específicos de los Estados miembros, de conformidad con la legislación o las prácticas nacionales, ni afectar al derecho a negociar, celebrar y aplicar convenios colectivos, o a emprender acciones colectivas de acuerdo con el Derecho o las prácticas nacionales. No se acierta a comprender que la robotización afecte a esos derechos concretos y específicos mencionados, derechos fundamentales colectivos de los trabajadores (negociación y conflicto colectivo), aun 
cuando la propuesta relativa al impacto positivo y a la prevención contra la afectación al ejercicio de los derechos fundamentales de las personas trabajadoras, sean individuales o colectivos, parece girar en torno a la idea o contexto tecnopesimista de sustitución de la mano de obra humana por robots, esto es, de eliminación o destrucción del empleo humano.

En este aspecto concreto, el de la sustitución de la mano de obra humana por robots (botsourcing en inglés), pensando en que fuera un efecto inevitable a corto plazo, cabe plantearse si la robótica se debe contemplar como un fenómeno tecnológico que pudiera incluirse entre los que pudieran generar discriminación en el disfrute de derechos y condiciones dignas de trabajo. Es por ello que se plantea articular en torno a la robótica una red de seguridad jurídica para los trabajadores, basada en el acervo normativo de la UE y en el nacional sobre el derecho a la no discriminación. Así, sería posible valorar y calificar jurídicamente no sólo los comportamientos arbitrarios de las personas empresarias, sino también aquellas conductas que pueden parecer formalmente como neutrales y vinculadas a un derecho empresarial de innovación tecnológico-robótica en la empresa. En caso de que, además, puedan verse afectados colectivos discriminados, entre otros, por factores como el género, la edad o la diversidad funcional, la robotización podría considerarse como una causa de discriminación indirecta (identificándose la robotización como un criterio neutro que tiene o puede tener un impacto adverso o resultar perjudicial para uno de los grupos vulnerables identificados para una de las causas en las que se prohíbe la discriminación) y activaría el proceso de garantía previsto en la normativa de la UE, especialmente en la Directiva 2000/78, de 27 de noviembre, relativa la establecimiento de un marco general para la igualdad de trato en el empleo y en la ocupación ${ }^{15}$.

En el ámbito del Derecho de la UE comienza a plantearse seriamente el derecho a la no discriminación algorítmica y, por tanto, cabría también formularlo desde la robótica, cuando lleva incorporada sistemas de IA. Se avanza hoy en propuestas de regulación de los algoritmos con fundamento en la transparencia y la inteligibilidad, garantizando el acceso a la información tanto a los y las trabajadoras como a sus re- presentantes colectivos si vulnerar los derechos de la empresa a la protección de su estrategia y patrimonio empresarial (tal y como se expresa en la Propuesta de Reglamento del Parlamento Europeo y del Consejo por el que se establecen normas armonizadas en materia de inteligencia artificial (ley de inteligencia artificial) y se modifican determinados actos legislativos de la Unión COM/2021/206 final ${ }^{16}$ y en la Propuestas de la Comisión para mejorar las condiciones laborales de las personas que trabajan a través de plataformas digitales ${ }^{17}$ ). Aspecto éste, por cierto, ya ampliamente tratado en el ámbito del Derecho del Trabajo, que procura la garantía de un resultado equilibrado entre los intereses de las personas empresarias y trabajadoras.

Así, por citar algún ejemplo, las causas o razones técnicas introducen en el sistema de relaciones laborales importantes dosis de flexibilidad en la gestión empresarial, interna y externa, tales como modificaciones sustanciales de las condiciones de trabajo, reducciones de jornada, suspensiones del contrato de trabajo o extinciones de la relación laboral. Esto nos lleva de inmediato a analizar la causa técnica-robótica con la propuesta de que se diseñen medidas orientadas a la igualdad retributiva, la aplicación efectiva y real del derecho a la igualdad de retribución por trabajo de igual valor, en este caso trabajo humano de igual valor al del robot, y medidas relacionadas con la eliminación de los puestos vacantes (o los despidos o cambios de trabajo) derivados de la robotización.

Más allá de la repercusión mediática que esta cuestión ha suscitado en nuestro país, y de apreciaciones no exactamente contrastadas en resoluciones judiciales del Orden Social de la Jurisdicción, en el actual marco normativo español, como bien ha expresado la doctrina, "cuando se trata de justificar despidos vinculados a la introducción de cambios tecnológicos en la empresa, no cabe exigir que tales cambios obedezcan a problemas o dificultades ya constatadas, ni tampoco acreditar nada acerca de la repercusión más o menos positiva de dichos cambios en la viabilidad o evolución de la empresa» (Jurado Segovia, 2020: 22).

Aunque las reglas normativas actuales no estaban pensando en el avance fulgurante de la innovación tecnológica-robótica con IA, más allá del sector industrial, la cuestión que se plantea ahora, a nuestro juicio

15 Disponible en la Propuesta de Reglamento del Parlamento Europeo y del Consejo por el que se establecen normas armonizadas en materia de Inteligencia Artificial. Véase nota 16

16 Disponible en: https://eur-lex.europa.eu/legal-content/ES/TXT/?uri=CELEX:52021PC0206

17 Disponible en: https://ec.europa.eu/commission/presscorner/detail/es/ip_21_6605 
más profunda, impide orientarse hacia una disyuntiva falaz entre la libertad de empresa (reconocida como derecho en la Constitución Española, art.38) y la competitividad empresarial, de un lado, y el derecho al trabajo y el mantenimiento del empleo, por otro; y que se pretenda justificar, sin mayores datos contrastados, que la robotización y automatización pueden destruir un notable volumen de empleo, de modo que conduzca a una reinterpretación de la causa técnica que la circunscriba a las empresas con dificultades. Se comparta o no esta visión sobre los efectos de la automatización en el mercado de trabajo, es conveniente que quien legisle adopte una valoración o decisión equilibrada y actualizada sobre esa causa técnica-robótica y que especifique, a nivel normativo, la razón técnica derivada de la robotización, articulando medidas que limiten el impacto del despido (necesidad de medidas preventivas, previas al cese, y medidas correctoras inmediatas, necesidad de recolocar al trabajador afectado).

En torno a las medidas preventivas, una de las cuestiones que se plantean al respecto es la definición de ajuste razonable en cuanto a la necesaria readaptación del puesto de trabajo del trabajador sustituido por robots con el consiguiente problema de si estos ajustes pueden considerarse una carga excesiva para el empresario. En nuestra opinión habría que valorar la razonabilidad o no del ajuste en relación con los incentivos fiscales y las subvenciones a la innovación que haya o deban ofrecerse a los empresarios.

Por otro lado, se hace mención en los documentos de la UE al lugar del trabajo, sin mencionar si se trata de un lugar físico y si es el lugar o centro de trabajo (en terminología jurídico-laboral). Este hecho exige plantear medidas orientadas hacia la cooperación-colaboración-interactuación entre trabajadores y robots. Inmediatamente surgen aquí impactos positivos y adversos.

Hablemos entonces de las implicaciones de la robótica en la seguridad en el trabajo y abordemos con urgencia los retos que el derecho, en particular el de prevención de riesgos laborales, debe afrontar en materia de seguridad y salud en el trabajo colaborativo entre humanos-robots. Se ha de intervenir en el marco de la prevención de los nuevos riesgos psicosociales como consecuencia de la presencia continua del robot alrededor de la persona, la carga mental originada por el miedo al contacto con el robot o el estrés causado por un número repetido de contactos entre el robot y la persona (en la fase de determinación, evaluación y planificación) atendiendo al principio de precaución o de cautela, mencionado en el art.191 del Tratado de Funcionamiento de la Unión Europea ${ }^{18}$. Su ámbito de aplicación es más amplio que el de la protección del medio ambiente y, como bien ha advertido Belén Muñoz Ruiz (2020), la doctrina se extiende al ámbito de la prevención de riesgos laborales más aún desde la perspectiva de los riesgos de la digitalización. De modo que parece oportuno, en estos momentos, revisar y actualizar la Comunicación de la Comisión sobre el recurso al principio de precaución, de 1 de febrero de $2000^{19}$.

Durante la primera etapa de transición, para asegurar una conversión responsable hacia un nuevo modelo de producción, han de valorarse seriamente las acciones públicas tendentes a procurar a los empresarios incentivos, incluso financieros, para el reciclaje y reubicación de trabajadores y adaptabilidad tecnológica.

\subsection{Formación y educación para (y en) el empleo.}

Si analizada la formación en la fase inicial, o para el empleo, y en la posterior, formación y reciclaje o readaptación en el empleo, seguramente, la orientación más detallada se encuentra en la Resolución del Parlamento Europeo, de octubre de 2020, con recomendaciones destinadas a la Comisión sobre un marco de los aspectos éticos de la inteligencia artificial, la robótica y las tecnologías conexas (véase nota 8) que:

-Advierte de la importancia de la inversión empresarial en la formación formal e informal y en el aprendizaje permanente, a fin de apoyar una transición justa hacia la economía digital.

-Destaca, en este contexto, que las empresas tienen la responsabilidad de garantizar un reciclaje profesional y una mejora de las capacidades adecuados a todos los empleados afectados, a fin de que aprendan a utilizar herramientas digitales y a trabajar con robots colaborativos y otras nuevas tecnologías, adaptándose así a las necesidades cambiantes del mercado laboral y conservando el empleo.

Se resume en aprendizaje permanente, reciclaje profesional y mejora de las capacidades. En esta

18 Disponible en: https://eur-lex.europa.eu/legal-content/ES/ALL/?uri=celex\%3A12012E\%2FTXT

19 Disponible en: https://eur-lex.europa.eu/legal-content/ES/ALL/?uri=CELEX\%3A52000DC0001. 
resolución, el Parlamento Europeo pide expresamente a los Estados miembros que «inviertan en sistemas de educación, formación profesional y aprendizaje permanente de alta calidad, adaptables e inclusivos, así como en políticas de reciclaje profesional y de mejora de las capacidades para los trabajadores de sectores que puedan verse gravemente afectados por la inteligencia artificial; destaca la necesidad de dotar a la mano de obra actual y futura de las capacidades necesarias en lectura, escritura, cálculo y competencias digitales, así como de competencias en ciencias, tecnología, ingeniería y matemáticas (CTIM) y de competencias interpersonales transversales, como el pensamiento crítico, la creatividad y el emprendimiento; subraya que, en este contexto, debe prestarse especial atención a la inclusión de los grupos desfavorecidos».

Hace tiempo que señalamos la necesidad de definir un marco legal y económico para facilitar el período de transición, tomando en cuenta los cambios en el aprendizaje de la población trabajadora para adaptarse a los nuevos trabajos y tareas. En un reciente trabajo mencionamos: "Como las previsiones relacionadas con los riesgos de desplazamiento de trabajadores en una empresa pueden variar con el paso del tiempo, debido a la velocidad del cambio tecnológico y a las mejoras de la robótica dotada de sistemas de inteligencia artificial, una empresa debe considerar la posibilidad de transferir a un tercero el riesgo probable de desplazamiento de trabajadores en el futuro» (López Sánchez, Grau Ruiz y Sánchez-Urán Azaña, 2019:40). Y planteábamos diferentes situaciones que, en el fondo, son las que ahora también parecen requerir las instituciones de la UE. En concreto, destacábamos las tres siguientes:

a) La falta temporal de competencias puede llevar a reforzar la inversión en formación durante la fase de transición, mientras el trabajador no puede trabajar.

b) Cuando la recapacitación no es factible por circunstancias especiales de vulnerabilidad, y es necesaria una red de seguridad.

c) Cuando la compatibilidad entre la formación y el trabajo es posible, por ejemplo, en la colaboración con robots interactivos, o cobots, la adaptación humana permite el reciclaje o el establecimiento de nuevos contratos. Esta situación es la óptima, porque el ser humano mantiene su puesto de trabajo, aprende haciendo, genera ingresos en lugar de demandarlos y evita el botsourcing, es decir, la sustitución de personas por robots.

Como también se ha afirmado, ante los avances tecnológicos «la formación es un valor estratégico para favorecer la productividad, la competitividad y el empleo» (García Piñeiro, 2020:180), de modo que la efectividad del derecho de los trabajadores a la formación profesional, reconocido en nuestra legislación laboral a través de varios mecanismos, requiere de la puesta en marcha inmediata de medidas reales y efectivas para responder adecuadamente a los retos de la digitalización en el marco de la política de empleo. Entre ellas cabe destacar la identificación como colectivo prioritario el de los trabajadores con déficits de formación en el ámbito de las nuevas tecnologías y digitalización; la introducción de incentivos económicos a las empresas, bien a través de mecanismos fiscales o de reducción o bonificación en las cotizaciones cuando la empresa invierta en formación y cualificación de sus trabajadores. Es el momento de incentivar la innovación tecnológica social y jurídicamente responsable orientada a garantizar los derechos de la población trabajadora. También de incentivar la formación, readaptación y recualificación. Pero no lo es, aunque haya ya propuestas y estudios gubernativos en marcha. En nuestro país, por ejemplo, el documento vigente en torno a la recomendación 20a sobre "Seguridad Social y Economía Digitalizada», es el Informe de evaluación y reforma del Pacto de Toledo de la Comisión de Seguimiento y Evaluación de los Acuerdos del Pacto de Toledo ${ }^{20}$, que contempla, solo desde una perspectiva cortoplacista, el objetivo de la recaudación a través de impuestos o cotizaciones por el uso de robots o por la inversión de las empresas en innovación tecnológica.

\section{AGRADECIMIENTOS}

Este estudio se ha realizado en el marco de los Proyectos INBOTS, CSA UE Proyecto 780073: Inclusive Robotics for a better Society; y FurWorTech, PID2019104287RB-100: "El impacto de la digitalización en las relaciones de trabajo: retos y oportunidades". Nuestro agradecimiento a los IPs y miembros de los equipos de investigación de ambos proyectos por sus reflexiones y consideraciones.

20 Puede consultarse en el Boletín Oficial de las Cortes Generales: https://www.congreso.es/public_oficiales/L14/CONG/BOCG/D/BOCG-14-D-175.PDF 


\section{BIBLIOGRAFÍA}

Álvarez Cuesta, Henar (2020). El impacto de la inteligencia artificial en el trabajo: desafios y propuestas. Navarra: Editorial Aranzadi.

Comisión Europea (2020). Libro Blanco sobre la inteligencia artificial: un enfoque europeo orientado a la excelencia y la confianza. COM (2020) 65 final. Disponible en: https:// ec.europa.eu/info/sites/default/files/ commission-white-paper-artificialintelligence-feb2020_es.pdf

De Asís, Rafael (2014). Una mirada a la Robótica desde los derechos humanos. Madrid: Dykinson.

Díaz, Alfredo y Grau Ruiz, María Amparo (2020). Social Security and robotization: Possible ways to finance human reskilling and promote employment. Paladyn, Journal of Behavioral Robotics, 11: 340-350. https://doi.org/10.1515/ pjbr-2020-0020

García Piñeiro, Nuria (2020). Política de Empleo y Nuevas Tecnologías. En Erik Monreal, Javier Thibault y Ángel Jurado (coords): Derecho del Trabajo y Nuevas Tecnologías. Valencia: Tirant lo blanch, pp.163-187.

García-Prieto Cuesta, Juan (2018). ¿Qué es un robot? En: Moisés Barrio Andrés: Derecho de los Robots. Madrid: Wolters Kluwer, pp. 29-64.

Grupo Independiente de Expertos de alto nivel sobre IA (2019). Directrices éticas para una IA Fiable. Bruselas: Comisión Europea. Disponible en: https://urjes.com/ pruebas/wp-content/uploads/2021/05/ Grupo-independiente-de-expertos-...Directrices-eticas-para-una-inteligenciaartificial-IA-fiable-ES.pdf.

Jurado Segovia, Ángel (2020). Robotización/ automatización y despido objetivo por causas técnicas (Art. 52 c ET). Revista Labos, 1 (3): 13-35. Disponible en: https://e-revistas.uc3m.es/index.php/
LABOS/article/view/5771/4126 https:// doi.org/10.20318/labos.2020.5771

Lin, Patrick; Abney, Keith y Bekey George (2011). Robot Ethics: Mapping the issues for a mechanized world. Artificial Intelligence, 175 (5-6): 942949. Disponible en: https://core.ac.uk/ download/pdf/19157493.pdf. https:// doi.org/10.1016/j.artint.2010.11.026

López Sánchez, José Ignacio; Grau Ruiz, María Amparo y Sánchez-Urán Azaña, María Yolanda (2019). The impact of robotics and computerization on the labor market: Inclusive Insight from a Law and Economics Perspective. Revista Derecho Digital e Innovación, 3: 30-60.

López-Sánchez, Jose Ignacio; ArroyoBarrigüete, Jose Luis y Curto-González, Tomás (2020). Automation and inequality. UCJC Business and Society Review, 17 (4): 148-176.

Lund, Susan; Madgavkar, Anu; Manyika, James; Smit, Sven; Ellingrud, Kweilin y Meaney, Mary (2021). The Future of Work after COVID-19. Mckinsey Global Institute web. Disponible en: https://www.mckinsey.com/featuredinsights/future-of-work/the-futureof-work-after-covid- [última consulta: septiembre 2021].

Montoya Melgar, Alfredo (2020). La dignidad del trabajo. Lección magistral con motivo de sus cincuenta años de Catedrático de Derecho del Trabajo. Murcia: Universidad de Murcia.

Muñoz Ruiz, Belén (2020). Robótica y Derecho del Trabajo: una revisión de los primeros fallos judiciales. Revista Trabajo y Derecho, 62: 67-77.

Organización Internacional del Trabajo (2019). Trabajar para un futuro más prometedor. Ginebra: Oficina Internacional del Trabajo. Disponible en: https://www.ilo.org/ wcmsp5/groups/public/---dgreports/--cabinet/documents/publication/ wcms_662442.pdf.
Palmerini, Erica (2017). Robótica y derecho: sugerencias, confluencias, evoluciones en el marco de una investigación europea. Revista de Derecho Privado, 32: 53-97. Disponible en: https://revistas. uexternado.edu.co/index.php/derpri/ article/view/5021/6009 https://doi. org/10.18601/01234366.n32.03

Parlamento Europeo (2017). Informe del Parlamento Europeo con recomendaciones destinadasalaComisión sobre normas de Derecho civil sobre robótica (2015/2103(INL)). Disponible en: https://www.europarl.europa.eu/doceo/ document/A-8-2017-0005_ES.html.

Parlamento Europeo (2020). Informe con recomendaciones destinadas a la Comisión sobre un régimen de responsabilidad civil en materia de inteligencia artificial. Disponible en: https://www.europarl.europa.eu/ doceo/document/A-9-2020-0178_ ES.html [Última actualización: 19 de octubre de 2020].

Veruggio, Gianmarco (2006). The EURON Roboethics Roadmap. En Giulio Sandini y Aude Billard: Proceedings of the 2006 6th IEEE-RAS International Conference on Humanoid Robots, December 4-6, 2006, Genoa (Italy), pp. 612-617. Disponible en https://www3.nd.edu/ rbarger/ethicsroadmap.pdf https://doi.org/10.1109/ ICHR.2006.321337

Veruggio, Gianmarco y Operto, Fiorella (2006). Roboethics: a bottom-up interdisciplinary discourse in the field of applied ethics in robotics. International Review of Information Ethics, 6 (12): 2-9. https://doi. org/10.4324/9781003074991-9

Verruggio, Gianmarco y Operto, Fiorella (2008). Roboethics: Social and Ethical Implications of Robotics. En Bruno Sicilianoy Oussama Khatib (eds.): Springer Handbook of Robotics. Suiza: Springer International Publishing, pp. 1499-1524. https://doi.org/10.1007/978-3-54030301-5_65 